\title{
Stephen Hawking, étudiant à Cambridge
}

D'après un entretien avec Brandon Carter, propos recueillis le 29 mars 2018 par Christiane Vilain, membre du comité de rédaction de Reflets de la physique.

En interrogeant Brandon Carter,

j'espère échapper à l'image

standard du " génie-grand-malade »,

véhiculée par les médias après

le décès de Stephen Hawking

en mars 2018, et même bien

avant, depuis la publication

de son livre intitulé Une brève

histoire du temps, en 1988.

Carter a fait ses études

universitaires au Pembroke

College de Cambridge.

Après son travail de thèse

et en postdoc dont il est

question dans le texte qui suit,

il est venu vivre en France pour

raisons personnelles ; il a alors

intégré le GAR (Groupe

d'Astrophysique Relativiste)

récemment créé à l'Observatoire

de Meudon, et a beaucoup

contribué au développement

de ce groupe devenu ensuite

le DARC, intégré aujourd'hui

au LUTH du même établissement.

Carter y est aujourd'hui Directeur

de recherche émérite.
Certes, Hawking était très doué : «Je n'ai pu obtenir des résultats personnels à propos des trous noirs et avoir une antériorite sur Stephen à ce sujet, que parce qu'il ne s'y intéressait pas encore! » dit Brandon Carter. "Il était vraiment plus fort que moi ! Heureusement pour ma carrière, il avait commencé par s'intéresser au problème du big-bang en cosmologie, et non aux trous noirs. " Hawking était par ailleurs déjà assez malade : "Je l'aidais souvent à rentrer chez lui, car il n'était pas encore en fauteuil, mais devait se tenir à quelque chose ou quelqu'un pour marcher " dit encore Brandon.

Mais ces deux caractéristiques de Stephen Hawking, qui donnèrent plus tard l'image d'un être étrange, presque extra-terrestre, oracle qui statue depuis son fauteuil sur l'origine et l'avenir du monde grâce à un appareillage sophistiqué capable de traduire en paroles les mouvements d'un muscle de sa joue, ces fantasmes ne nous donnent pas accès à sa personne même. Reste son humanité souffrante, son intelligence aiguë appuyée sur beaucoup de travail et l'évolution de ses intérêts.

\section{Les étudiants de Denis Sciama à Cambridge}

Stephen Hawking était en thèse avec Denis Sciama, astrophysicien à Cambridge, depuis un an, et Georges Ellis ${ }^{(a)}$ depuis deux ans, lorsque Carter a débuté en 1962 sa thèse avec ce même tuteur, en même temps que Martin Rees ${ }^{(b)}$. Ce dernier aujourd'hui Lord Rees - était le seul à être astrophysicien comme Denis Sciama et à savoir précisément ce qu'il voulait faire. Les trois autres s'intéressaient à la pure physique théorique, d'une façon plus générale. Hawking était d'abord très mathématicien, mais s'était orienté vers l'astronomie - et donc vers la physique lors de ses études à Oxford.

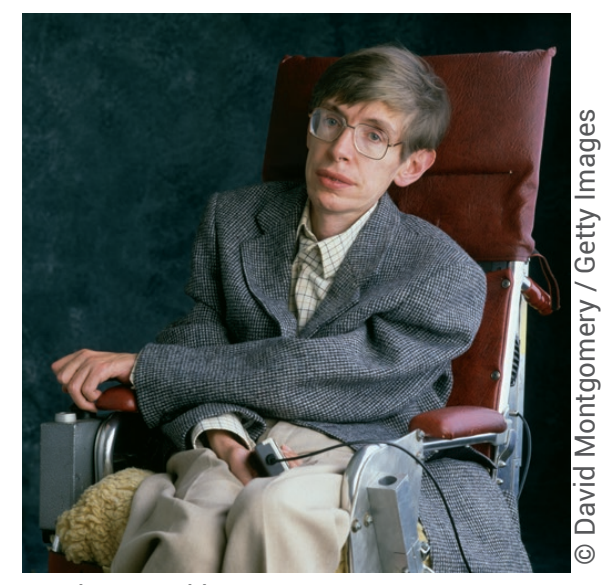

Stephen Hawking

Sciama a en conséquence rapidement adressé ses deux élèves, Carter et Hawking, à Roger Penrose qui avait environ sept ans de plus qu'eux et travaillait à Londres. Ils se rendaient ainsi chaque semaine en train à Londres pour parler avec lui, souvent à l'occasion du séminaire qui avait lieu au King's college londonien, sous la direction de Hermann Bondi.

En cosmologie théorique, on se demandait si la singularité initiale dans la solution des équations d'Einstein ne pouvait pas être due à l'homogénéité supposée de la matière de l'Univers, et si elle ne disparaitrait pas dans des conditions plus réalistes. Penrose a aidé Hawking à travailler sur la question en utilisant d'autres équations que celles des solutions exactes, et le résultat a été que la singularité était inévitable, quoique sous une forme plus compliquée qu'une simple densité infinie en un point.

Hawking et Penrose n'étaient pas les seuls à chercher à éviter cette singularité, que Fred Hoyle avait nommée par dérision : "Big Bang". Hoyle - connu également pour ses romans de science-fiction comme Le nuage noir - proposait d'en rester à un Univers à densité stationnaire, avec création continue pour compenser l'expansion attestée par les décalages vers le rouge. 


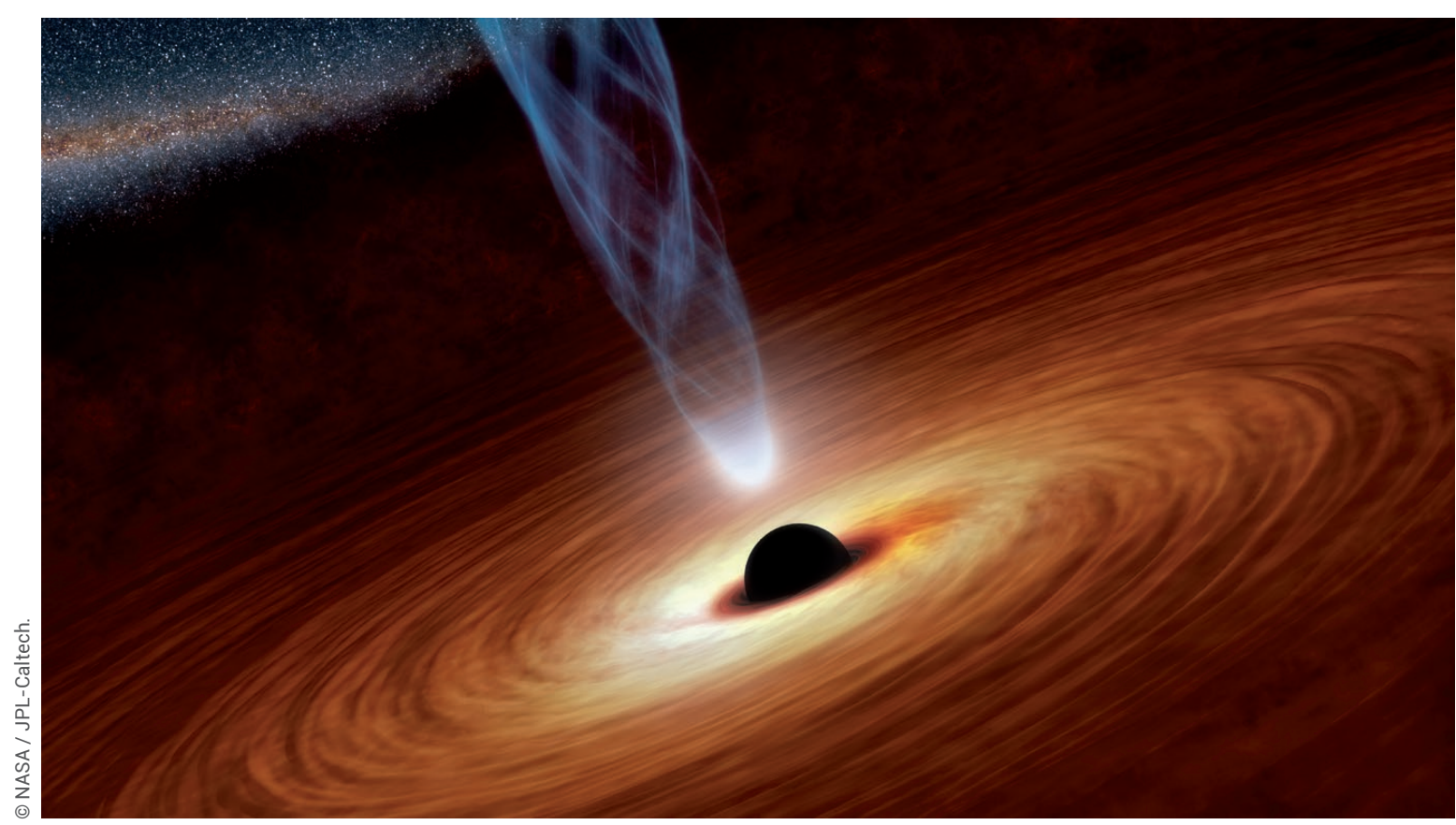

Vue d'artiste d'un trou noir supermassif, entouré d'un disque d'accrétion dont la région centrale émet un jet de rayons $X$ de haute énergie.

Cette théorie était défendue également par Bondi et Tommy Gold. Le trio travaillait ensemble depuis 1942, mais Hoyle réussit à mettre au point au début des années 1960 une formulation plus précise et fit à Londres en 1963 une conférence à ce sujet. L'étudiant Stephen Hawking était là et lui dit à un moment : " Mais savez-vous que cette intégrale diverge ? " Hoyle lui demanda comment il le savait : « je l'ai calculée " répondit Stephen ; ce fut la fin de cette première phase de l'aventure connue ensuite sous le nom de "Steady State" et poursuivie par Hoyle et Jayant Narlikar jusqu'à la découverte du rayonnement à $3 \mathrm{~K}$ en 1964.

Hawking a soutenu en 1966 son $P h D$ sur les propriétés de l'Univers en expansion. Il publiera en 1973 avec Ellis le livre de

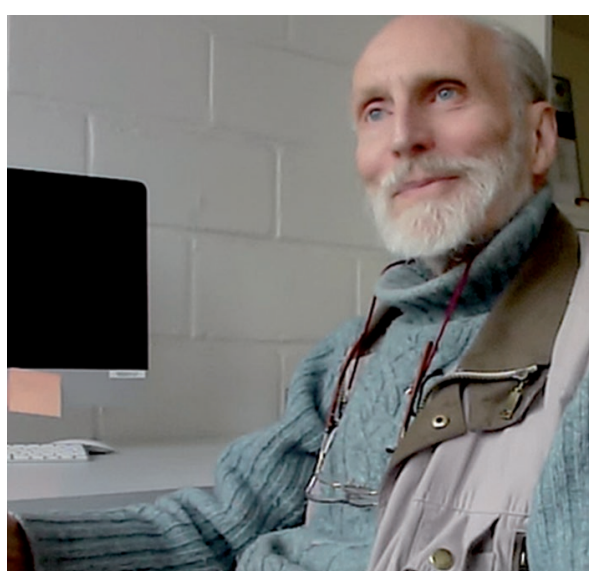

Brandon Carter relativité générale intitulé : The large scale structure of space-time, et beaucoup plus tard en 1996 avec Roger Penrose le livre de vulgarisation: The nature of space and time.

\section{Les trous noirs de Kerr}

Pendant cette même période des années 1960 à Cambridge, Penrose avait mis au point une projection particulière de l'ensemble des géodésiques de l'espace-temps de Minkowski qui, comme les projections de Mercator pour la Terre, a la particularité de conserver les angles; alors les géodésiques lumière se croisent toujours avec le bon angle sur le diagramme.

Carter avait besoin de généraliser ces diagrammes pour étudier une nouvelle solution des équations d'Einstein publiée en 1963 par Roy Kerr, mathématicien néo-zélandais, solution à symétrie axiale pour un corps en rotation qui complète la solution établie pour un corps statique à symétrie sphérique par Karl Schwarzschild en 1915, l'année même de la publication par Einstein des équations de la relativité générale.

C'est cette solution qui devait donner lieu à notre concept de " trou noir ", selon le nom introduit ensuite par John Archibald Wheeler. Les diagrammes établis pour les solutions de Minkowski, de Schwarzschild et de Kerr sont rangés aujourd'hui sous le nom général de "diagrammes de CarterPenrose ».
Un trou noir est caractérisé par son horizon ou "event horizon", surface à partir de laquelle la lumière elle-même ne peut s'échapper, interdisant toute communication entre l'extérieur et un intérieur qui demeure mystérieux.

L'horizon doit être distingué de l'ergosurface (fig. 1) sur laquelle le vecteur générateur de la symétrie stationnaire ${ }^{(c)}$, qui est normalement de "genre temps » $(d)$ devient de "genre espace "; plus concrètement, c'est aussi la surface à partir de laquelle un corps extérieur quelconque ne peut plus demeurer stationnaire, entraîné par la rotation du trou noir sans être encore à ce stade entraîné vers le centre. Dans le cas statique de Schwarzschild, l'ergosurface est confondue avec l'« horizonévénement " et ne peut donc être le lieu d'aucun phénomène observable.

Carter démontra également en 1968 l'intégrabilité des équations des géodésiques dans la solution de Kerr, qui n'était pas évidente puisque cette solution n'admet que deux constantes du mouvement, associées aux deux symétries : stationnarité et symétrie axiale ; il lui a fallu en trouver deux autres pour résoudre le système. L'une était facile et l'autre, plus délicate, est appelée aujourd'hui la " constante de Carter ».

Brandon Carter rappelle : "Vers la fin des années 1960, Stephen commença à se rendre compte que les trous noirs étaient plus excitants que le Big-Bang ! » et il fut 
le premier à examiner la difficile question de savoir si un trou noir en rotation stationnaire doit forcément être à symétrie axiale, une condition qui n'avait rien d'évident puisque des solutions non symétriques existaient en mécanique newtonienne. Cette hypothèse - qui s'avéra juste par la suite - avait été posée au départ dans la démonstration de l'unicité de la solution de Kerr, effectuée par Brandon Carter et complétée par David Robinson.

Carter et Hawking continuèrent donc à travailler avec Penrose sur les trous noirs. Les équations de la solution de Kerr permettent des géodésiques fermées qui violent le principe de causalité, mais c'est sans conséquence physique puisque celles-ci se situent à l'intérieur de l'horizon, dans tout le domaine de paramètres qui donne lieu à un trou noir et qui sera seul utilisé pour rendre compte des observations.

\section{Thermodynamique du trou noir et rayonnement de Hawking}

Puis vint à Cambridge un chercheur américain nommé Jacob Bekenstein, qui avait mis au point une analogie thermodynamique pour les trous noirs, dont les caractéristiques se réduisaient à peu de choses : une masse et un rayon. Bekenstein proposait que la surface de l'horizon, dont Hawking avait montré qu'elle ne pouvait que croître, représente l'entropie du trou noir. Si cet objet a une entropie, il doit avoir également une température et celle-ci peut en effet être représentée par une quantité liée à l'accélération à la surface de l'horizon. Bekenstein avait publié en avril 1973 : "Black holes and entropy"; James Bardeen, Carter et Hawking ont publié ensemble en juin 1973 : "The four laws of black hole mechanics".

Mais un objet qui a une température doit rayonner ; et nous voilà maintenant au cœur du travail de Steven Hawking, à partir d'un paradoxe auquel il ne croit pas lui-même au départ. Rien ne peut sortir du trou noir, mais il doit rayonner. La solution émergea de la mécanique quantique avec des créations de paires au voisinage de l'horizon. Hawking effectua les calculs, que les autres n'étaient sans doute pas capables de faire ou insuffisamment motivés, et trouva à sa grande surprise que cela marchait. C'est là le fameux « rayonnement de Hawking ".

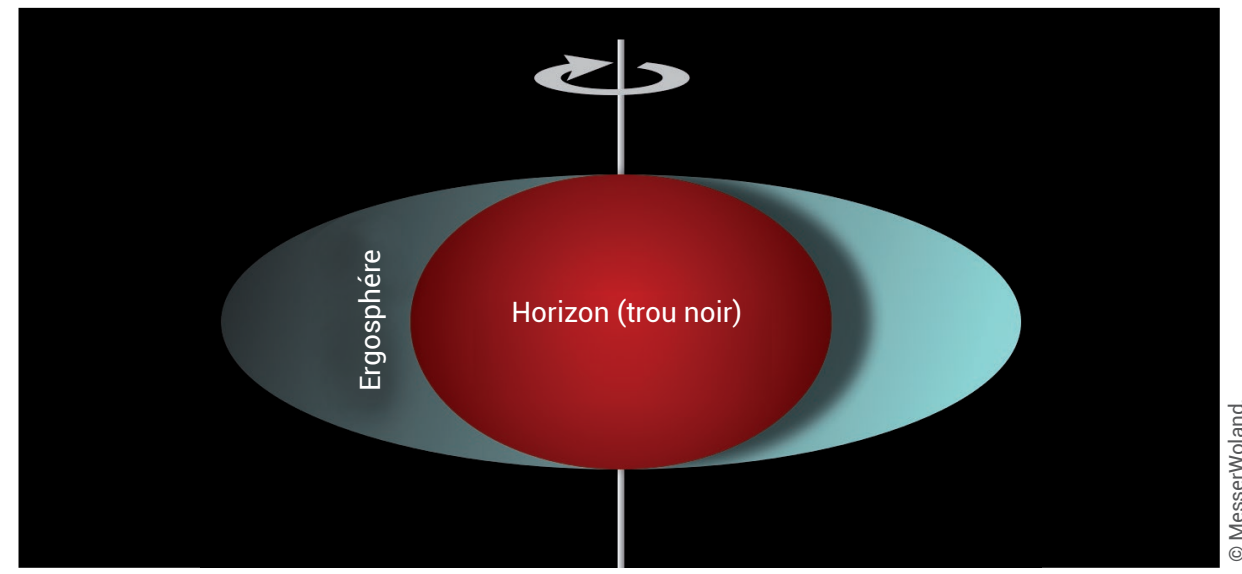

1. Horizon des événements et ergosphère d'un trou noir en rotation. Les particules passant à l'intérieur de l'ergosphère peuvent, dans certaines conditions, gagner de l'énergie mécanique au détriment du trou noir puis s'en échapper. Ce dernier perd alors du moment angulaire.

Les trous noir "s'évaporent " donc ! D'autant plus rapidement qu'ils sont plus petits. Et si, comme le proposa Hawking un peu plus tard, une grande quantité de ces minitrous noirs ont été créés au début de l'Univers, ils se sont évaporés et s'évaporent peut-être encore! Beaucoup de questions ont été soulevées ensuite à propos de l'éventuelle perte d'information qui résulterait de cette résurgence sous forme de simples photons de tout ce qui est tombé dans le trou noir..

\section{Ensuite...}

Hawking aurait précisé cependant vers la fin de sa vie, que l'on ne pourrait rien dire de déterminant à ce sujet (ni sans doute au sujet de l'évaporation) tant que l'on n'aurait pas une théorie unifiée de la gravitation et de la mécanique quantique. La physique quantique est non locale et sa théorie standard, qui fonctionne parfaitement dans nos conditions habituelles de faible courbure de l'espace, est en effet plaquée là sur un champ de gravitation généralement très fort et donc une forte courbure.

Hawking avait été élu en 1974 à la Royal Society, en dépit d'une aggravation de sa maladie qui détériora son élocution à partir des années 1970. Mais ce fut une pneumonie grave qui nécessita une trachéotomie et le priva définitivement de la parole en 1985. Il était revenu à ses premières amours pour la cosmologie, à partir de 1983 avec Jim Hartle, cherchant toujours à éviter ce début de l'Univers par un espace sans bord et un temps imaginaire, recherche qu'il aurait considéré à la fin comme la plus importante de sa vie.
L'étudiant de Cambridge est devenu plus tard - en dépit de son lourd handicap à son tour professeur, occupant la chaire lucasienne qui avait été celle de Newton et de Dirac à Cambridge, à partir de 1980.

Brandon Carter entretenait de bonnes relations avec la famille Hawking; il s'est rendu à l'enterrement à Cambridge le 31 mars, puis à l'inhumation des cendres de Stephen Hawking à l'abbaye de Westminster à Londres en juin.

(a) Le cosmologiste Georges Ellis est originaire de Johannesburg. Il est retourné en Afrique du Sud dans les années 1970 et est aujourd'hui professeur émérite à l'Université du Cap. Il a été nommé Docteur honoris causa de l'Université Pierre et Marie Curie de Paris en octobre 2016.

(b) Martin Rees est un astrophysicien bien connu pour son abondant travail sur la répartition et la formation des amas de galaxies, les quasars comme noyaux galactiques et enfin le fond diffus cosmologique. Étudiant au Trinity College en même temps que Carter, puis en thèse également avec Denis Sciama, il est devenu professeur d'astronomie au Gresham College en 1975, puis enseignant-chercheur pour la Royal Society, dont il devient président en 2005.

(c) À toute symétrie quelconque du système (une des coordonnées n'intervient pas dans les équations), on peut en effet associer un champ de vecteurs générateurs sur la variété espace-temps (appelé " champ de Killing »), ainsi qu'une loi de conservation (d'un flux). Ce dernier point avait été démontré par Emmy Noether dès 1915 et s'applique aussi bien hors du cadre de la relativité générale.

(d) Les géodésiques qui décrivent les trajectoires possibles de particules doivent correspondre à des vitesses inférieures à celle de la lumière ; elles sont dites " de genre temps " parce qu'elles se situent à l'intérieur du cône centré sur l'axe des temps (axe qui montre lui-même l'évolution d'un point immobile dans le repère choisi). Les directions extérieures à ce cône représentent des interactions à vitesse supérieure à celle de la lumière, et sont donc impossibles dans tout modèle qui respecte la causalité. 